\title{
The Relation of Mitral Valve Morphology to Severe Mitral Regurgitation Complicated With Mitral Valve Prolapse
}

\author{
Makoto Sonoda, MD*, Katsu Takenaka, MD**, Kansei Uno, MD***, \\ Aya Ebihara, MD** and Ryozo Nagai, MD* \\ *Department of Cardiovascular Medicine, University of Tokyo Graduate School of Medicine, \\ Tokyo, Japan \\ **Department of Laboratory Medicine, University of Tokyo Hospital, Tokyo, Japan \\ ***Computational Diagnostic Radiology and Preventive Medicine, University of Tokyo Graduate \\ School of Medicine, Tokyo, Japan
}

\begin{abstract}
Background. Severe mitral regurgitation (MR) is one of the most important complications of mitral valve prolapse (MVP) and it often requires surgical treatment. This study is aimed to assess the relation of mitral valve morphology to severe MR complicated with MVP.

Methods. Transesophageal echocardiography was performed in 37 patients with MVP and 30 control subjects.

Results. The anterior mitral leaflet (AML) and posterior mitral leaflet (PML) were thicker and longer, and the mitral annulus was larger in patients with MVP than in control subjects $(p<0.05)$. The degree of MVP correlated significantly with the leaflet thickness in systole (AML: $r=0.70, P M L: r=0.65, p<0.05$ ). In patients with MVP without ruptured chordae tendineae (RCT), the severity of MR correlated significantly with the leaflet thickness, leaflet length and annular diameter $(p<0.05)$. The prolapsed PMLs with RCT were thicker and longer than those without $R C T(p<0.05)$.

Conclusions. The leaflet thickness, leaflet length and annular diameter are "proportionally" redundant in patients with $M V P$, and the redundancy is closely related to the occurrence of RCT or severe MR.
\end{abstract}

(J Echocardiogr 2008; 6: 1-8)

Key words: mitral valve prolapse, mitral regurgitation, transesophageal echocardiography, Doppler ultrasound, ruptured chordae tendineae

\section{Introduction}

Mitral valve prolapse (MVP) is the most common abnormality of human heart valves, occurring in $\geq 3 \%$ of adults [1-2]. Severe mitral regurgitation (MR) is one of the most important complications of mitral valve prolapse [3-10] which may result from either progressive myxomatous degeneration or chordal rupture

Received August 8, 2007; revision received February 25, 2008; accepted February 25, 2008

Address for correspondence: Makoto Sonoda, MD

Department of Cardiovascular Medicine, University of Tokyo Graduate School of Medicine,

Hongo 7-3-1, Bunkyo-ku, Tokyo 113-8655, Japan.

Telephone: +81-3-3815-5411 (ext. 37161)

Fax: +81-3-3814-0021

E-mail: sonoda-2im@h.u-tokyo.ac.jp

(C) 2008 Japanese Society of Echocardiography
[10], for it often requires surgical treatment. The presence of ruptured chordae tendineae (RCT) is frequent in patients with mitral valve prolapse requiring surgical repair [11-15].

Two-dimensional echocardiography, the standard of cardiac ultrasound from the late 1970 s to the early 1980s [16], has become an important tool in the diagnosis of mitral valve prolapse because it allows direct visualization of the mitral leaflets. Transesophageal echocardiography, which has been established as a special field in echocardiography [17], can provide accurate evaluation of mitral valve prolapse and ruptured chordae tendineae.

This study was aimed to assess the relation of the mitral valve morphology to the severe mitral regurgitation complicated with mitral valve prolapse by transthoracic and transesophageal echocardiography. 


\section{Methods}

\section{Study subjects}

Transthoracic and transesophageal echocardiographic examinations were performed in 37 consecutive adult patients ( 21 men, 16 women, mean age $51.9 \pm 15.5$ years) given a diagnosis of mitral valve prolapse, and transesophageal echocardiography was performed in 30 sex- and age-matched control subjects (22 men, 8 women, mean age $54.3 \pm 12.3$ years). Written informed consent was obtained from all study subjects. Transthoracic echocardiographic examinations

For this procedure, all the subjects lay down in the left lateral decubitus position. Commercially available instruments (Toshiba, 380-A, ACUSON 128Xp) were used with a $2.5 \mathrm{MHz}$ phased array sector transducer. Under continuous monitoring of the limb lead II, twodimensional, pulsed wave Doppler and continuous wave Doppler studies were performed. The data were recorded on videotape.

\section{The diagnosis for mitral valve prolapse}

According to the previous report [18], mitral valve prolapse was defined as systolic displacement into the left atrium of one or both mitral leaflets in the parasternal long-axis view on two-dimensional echocardiography.

\section{Evaluation of the severity of mitral regurgitation}

The grading of the severity of mitral regurgitation was obtained by color-coded signal of mitral regurgita- tion. Mitral regurgitation was considered mild if turbulence was confined to the proximal third of the left atrium, moderate if turbulence was confined to the middle third of the left atrium and severe if turbulence extended into the distal third of the left atrium [19].

Transesophageal echocardiographic examinations

Under continuous monitoring of the limb lead II, transesophageal echocardiography was performed in 37 patients with MVP and 30 control subjects. A biplane or multiplane transesophageal probe (centerline carrier frequency $5 \mathrm{MHz}$ ) was used. Pharyngeal and hypopharyngeal topical anesthesia was induced with Lidocaine Viscous and Lidocaine Spray. Conscious sedation was not induced. Particular attention was directed toward complete visualization of the mitral valve.

\section{Evaluation of the degree of mitral valve prolapse}

According to the previous report [20], the area subtended by the line connecting the annular hinge points of the mitral leaflets and the coapted mitral leaflets were measured at their maximum in systole during transesophageal echocardiography (Figure 1). In this study, the degree of mitral valve prolapse was defined as this area indexed for body surface area.

\section{Ruptured chordae tentineae}

Ruptured chordae tendineae was diagnosed in patients with mitral valve prolapse when highly mobile echoes, compatible with ruptured chordal apparatus,

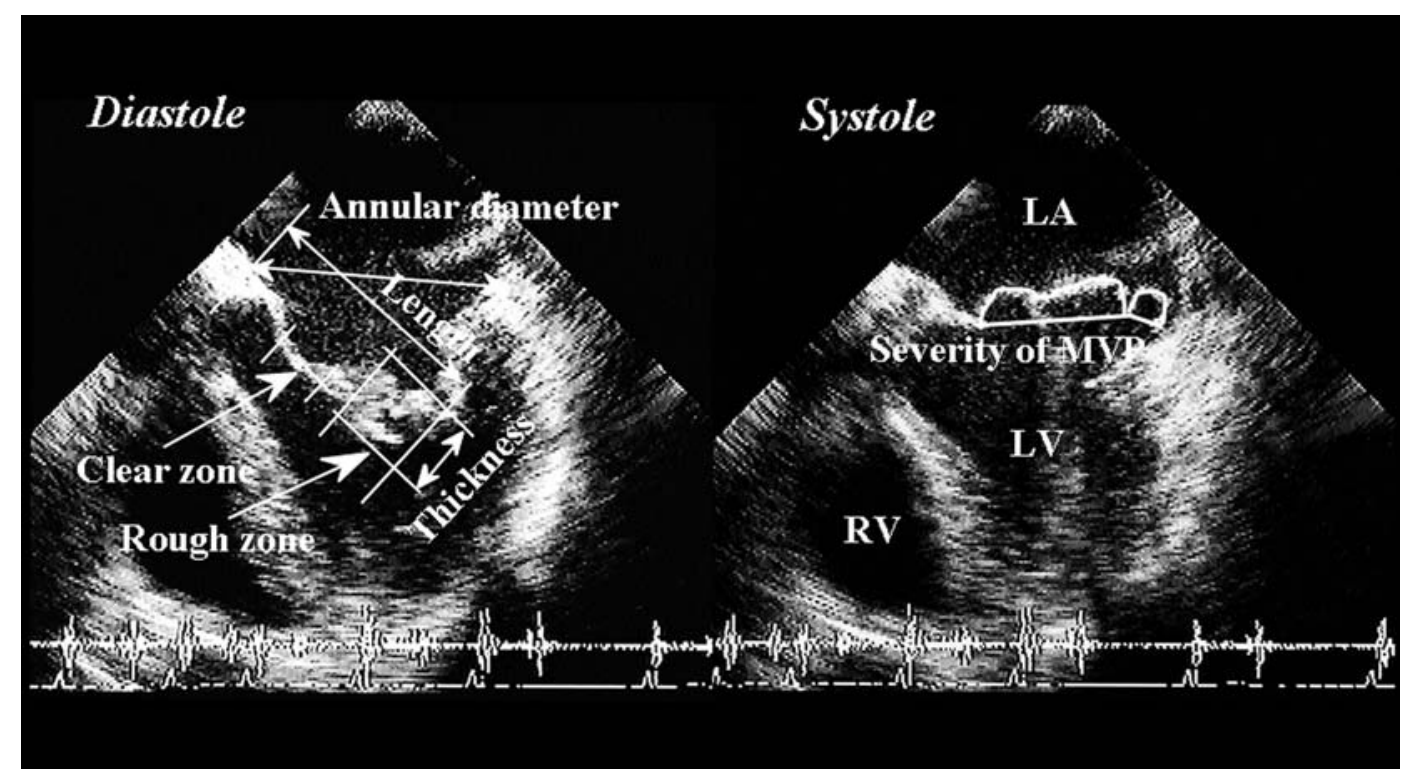

Fig. 1. Representative images of the heart obtained by transesophageal echocardiography from a retroatrial probe position in a patient with mitral valve prolapse. The four-chamber views of the left atrium (LA), right atrium, left ventricle (LV) and right ventricle $(R V)$ is illustrated in diastole (left) and systole (right). 
were seen prolapsing into the left atrium in systole and returning to the left ventricle in diastole.

\section{Mitral apparatus measurements}

Images of the mitral valve were fully examined in a transverse scan or longitudinal scan using a biplane transesophageal probe or in a view with the plane in the $0^{\circ}$ to $180^{\circ}$ position using multiplane transesophageal probe at any level of transesophageal probe insertion. Mitral apparatus measurements were obtained from images of the mitral valve in the transesophageal four-chamber view of the heart (Figure 1). The maximal leaflet thickness of rough zone (within the length of apposition) and clear zone (farther from the leaflet tip than the length of apposition) in diastole were measured as the distance from the ventricular to the atrial surface of the leaflet perpendicular to the surface of the leaflet. At the time of maximal leaflet prolapse, the prolapsed mitral leaflet thickness was measured at its portion displaced maximally into the left atrium. In mid to late systole, which corresponded to the timing of maximal systolic displacement of the prolapsed mitral leaflet into the left atrium, the leaflet thickness in control subjects was measured at the middle point between its hinge point and free edge. Mitral leaflet length was measured as the distance between its hinge point and free edge. Mitral annular diameter was assessed by measuring the length of the line connecting the anterior and posterior leaflet hinge points. It was measured at end-diastole (just before QRS complex) and mid-systole (approximating the time of maximal displacement of prolapsed mitral leaflets). All results in these measurements were indexed for body surface area.

\section{The "RATIO" and the " $r$-c RATIO"}

To assess the relation of the mitral annular dilatation to the mitral regurgitation complicated with mitral valve prolapse, we examined the "RATIO" defined as the ratio of mitral annular diameter in mid-systole to the sum of anterior and posterior mitral leaflet lengths. To assess the morphologic characteristics of the prolapsed mitral leaflets in detail, we examined the "r-c RATIO" defined as the ratio of mitral leaflet thickness of its rough zone to that of its clear zone in diastole.

\section{Reproducibility}

Inter-observer variability was determined from 10 measurements of anterior mitral leaflet thickness of rough zone in diastole taken by a second independent investigator. Intra-observer variability was determined from 10 re-measurements of anterior mitral leaflet thickness of rough zone in diastole following a 1-month interval. Data were obtained from simple linear regression analysis by the correlation coefficient $(r)$, and the significance of the relation (p).

\section{Statistical analysis}

Mean values are given with $1 \mathrm{SD}$. The differences between the two groups were compared by unpaired Student's $t$ test. Means were compared among three groups by means of analysis of variance with application of the Scheffe F test for significance. Association between mitral leaflet thickness and degree of mitral valve prolapse were made using Pearson's correlation coefficient. Relations between the severity of mitral regurgitation and leaflet thickness, leaflet length, annular diameter and the RATIO were made using Spearman's rank correlation. A p value $<0.05$ was considered statistically significant.

\section{Results}

\section{Mitral valve morphology in mitral valve prolapse}

As shown in Figure 2, the degree of mitral valve prolapse correlated significantly with the leaflet thickness in systole. It also correlated with the posterior leaflet length significantly $(r=0.47, p<0.05)$, but did not correlate with the anterior leaflet length $(r=0.43, p>0.05)$.

The echocardiographic data of patients with mitral valve prolapse and control subjects are summarized in Table 1. The mitral leaflet was thicker and longer; the mitral annulus was larger in patients with mitral valve prolapse than in the control subjects. The RATIO was similar between the two groups. The r-c RATIO was significantly larger in patients with mitral valve prolapse than in control subjects.

\section{Mitral regurgitation complicated with mitral valve prolapse}

As shown in Figure 3, in patients with mitral valve prolapse without ruptured chordae tendineae (open circle: mild mitral regurgitation: 7 patients, moderate mitral regurgitation: 7 patients, severe mitral regurgitation: 2 patients), the severity of mitral regurgitation correlated significantly with the anterior and posterior leaflet thickness, posterior leaflet length and annular diameter. It did not correlate with the anterior leaflet length and the RATIO. Similarly, in all patients with mitral valve prolapse, including the patients with ruptured chordae tendineae (closed circle: mild mitral regurgitation: 7 patients, moderate mitral regurgitation: 13 patients, severe mitral regurgitation: 17 patients), the results were the same. 


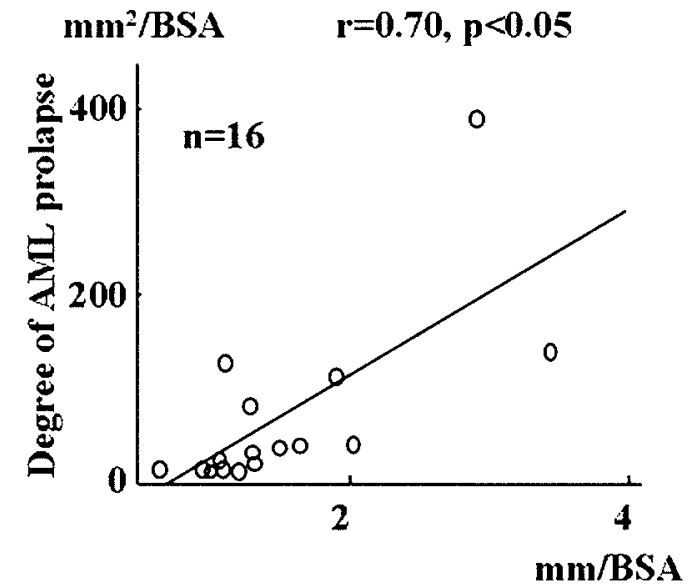

AML thickness in systole

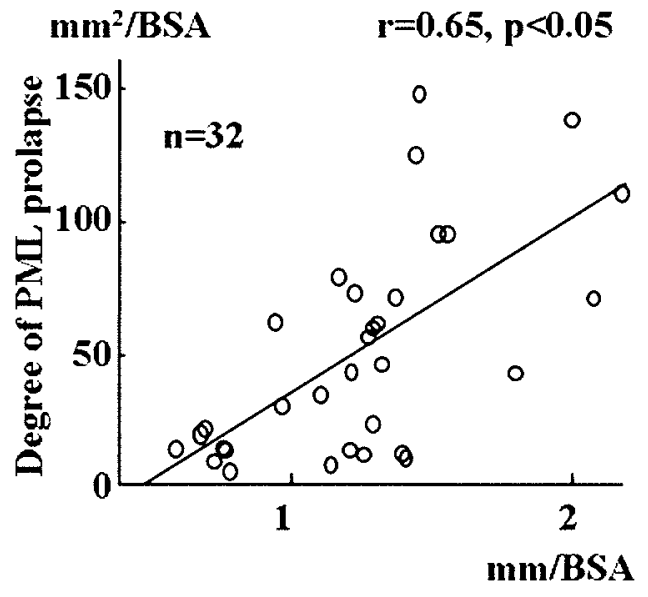

PML thickness in systole

Fig. 2. This figure shows the relationship between the mitral leaflet thickness in systole and the degree of MVP.

Table 1. The echocardiographic data of patients with mitral valve prolapse and control subjects.

\begin{tabular}{lrcc}
\hline & & MVP & Control subjects \\
\hline $\mathrm{n}$ & & 37 & 30 \\
AML thickness in diastole & $(\mathrm{mm} / \mathrm{BSA})$ & $1.92 \pm 0.64$ & $1.44 \pm 0.29^{*}$ \\
AML thickness in systole & $(\mathrm{mm} / \mathrm{BSA})$ & $1.39 \pm 0.54$ & $0.99 \pm 0.21^{*}$ \\
PML thickness in diastole & $(\mathrm{mm} / \mathrm{BSA})$ & $1.90 \pm 0.60$ & $1.39 \pm 0.29^{*}$ \\
PML thickness in systole & $(\mathrm{mm} / \mathrm{BSA})$ & $1.25 \pm 0.40$ & $0.97 \pm 0.24^{*}$ \\
AML length & $(\mathrm{mm} / \mathrm{BSA})$ & $16.6 \pm 4.3$ & $13.2 \pm 2.2^{*}$ \\
PML length & (mm/BSA) & $10.8 \pm 2.9$ & $7.8 \pm 2.2^{*}$ \\
Annular diameter in end-diastole & $(\mathrm{mm} / \mathrm{BSA})$ & $24.5 \pm 5.4$ & $18.4 \pm 2.8^{*}$ \\
Annular diameter in mid-systole & $(\mathrm{mm} / \mathrm{BSA})$ & $21.6 \pm 5.3$ & $16.3 \pm 2.5^{*}$ \\
RATIO & & $79.3 \pm 14.3$ & $78.6 \pm 12.5$ \\
r-c RATIO & & $1.83 \pm 0.34$ & $1.66 \pm 0.32^{*}$ \\
& AML & $1.63 \pm 0.35$ & $1.46 \pm 0.29^{*}$ \\
\hline
\end{tabular}

*:p $<0.05$ by unpaired student $t$ test

\section{Ruptured chordae tendineae}

The prolapsed posterior mitral leaflets with ruptured chordae tendineae were thicker and longer than those without ruptured chordae tendineae and those of the control subjects (Table 2).

\section{Reproducibility}

Inter-observer variability and intra-observer variability were satisfactory: $\mathrm{r}=0.93, \mathrm{p}<0.05$ for inter-observer viability and $r=0.96, p<0.05$ for intra-observer variability.

\section{Discussions}

\section{"Proportionally" redundant mitral valve}

Thicker mitral leaflets in patients with mitral valve prolapse evaluated by means of transthoracic echo [2127] or transesophageal echo [28-30] have already been reported in the previous studies. This is the first report that the mitral valve morphology was related to the severity of mitral regurgitation complicated with mitral valve prolapse using echocardiography.

Mitral valve prolapse was associated with myxomatous mitral valve [7, 21, 31-35]. In this study, the patients with mitral valve prolapse had thicker and longer mitral leaflets than control subjects. This finding may reflect the histological abnormalities of disproportionate enlargement of connective tissue elements of the mitral valve compared with their supporting fibrous elements in patients with mitral valve prolapse.

The RATIO was similar between patients with mitral valve prolapse and the control subjects. The mitral annulus was larger and the mitral leaflet was longer in patients with mitral valve prolapse than in control sub- 


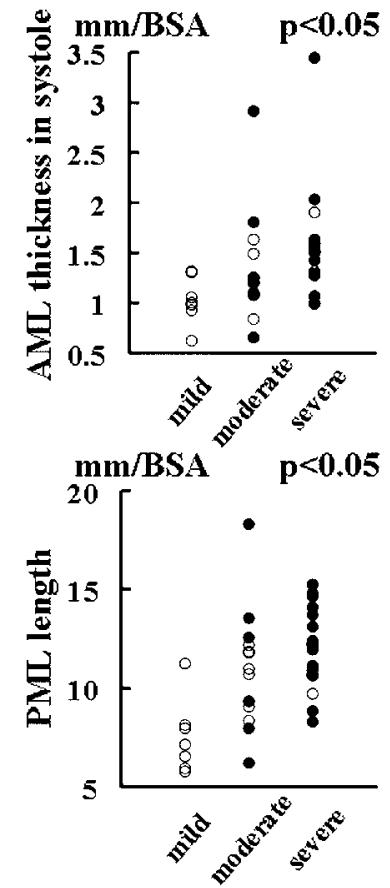

Severity of MR

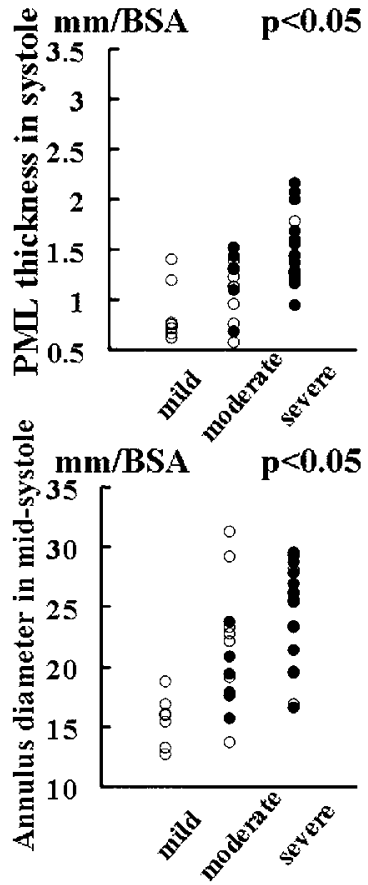

Severity of MR

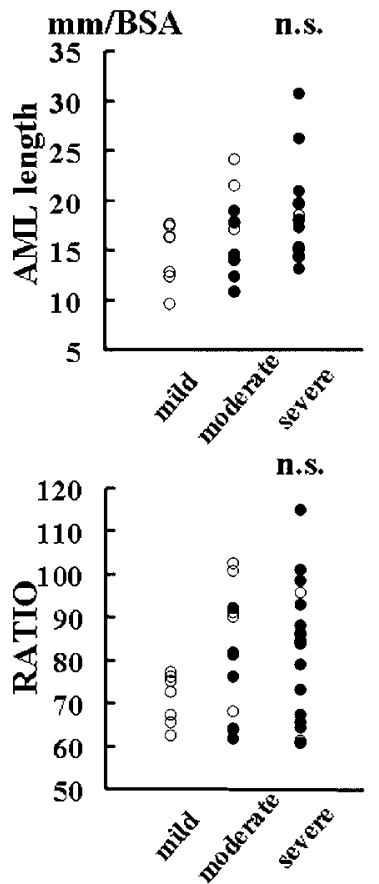

Severity of MR

Fig. 3. This figure shows the relationship between the severity of mitral regurgitation and the morphology of the mitral valve in patients with mitral prolapse without ruptured chordae tendineae (open circle) or with ruptured chordae tendineae (closed circle).

Table 2. The echocardiographic data of the prolapsed PMLs with RCT, prolapsed PMLs without RCT and PMLs of control subjects.

\begin{tabular}{|c|c|c|c|}
\hline & \multicolumn{2}{|c|}{ Prolapsed PMLs } & \multirow{2}{*}{ PMLs of control subjects } \\
\hline & with RCT & without RCT & \\
\hline $\mathrm{n}$ & 17 & 14 & 30 \\
\hline Thickness in diastole (mm/BSA) & $2.17 \pm 0.58^{*}$ & $1.61 \pm 0.53$ & $1.39 \pm 0.29$ \\
\hline Thickness in systole (mm/BSA) & $1.41 \pm 0.39 *$ & $1.04 \pm 0.35$ & $0.97 \pm 0.24$ \\
\hline Length $\quad(\mathrm{mm} / \mathrm{BSA})$ & $11.75 \pm 3.06^{*}$ & $9.23 \pm 2.17$ & $7.81 \pm 2.17$ \\
\hline
\end{tabular}

*:p $<0.05$ by the Scheffe $\mathrm{F}$ test

jects. These findings may be the reason why the RATIO did not differ between the two groups.

The r-c RATIO in patients with mitral valve prolapse has never been evaluated. This is the first report that the r-c RATIO was evaluated to assess the morphologic characteristics of the prolapsed mitral leaflets. It was larger in patients with mitral valve prolapse than in control subjects. This result was compatible to the histological finding that myxomatous degeneration existed mainly in the spongiosa layer of rough zone in prolapsed mitral leaflets [21, 33].
The present study confirms mitral leaflet thickness and length and mitral annular diameter are "proportionally" redundant in patients with mitral valve prolapse. Moreover, leaflet thickening was thought to be related pathologically to proliferation of myxomatous tissue in the spongiosa layer of the prolapsed leaflets, and this finding indicates that thicker mitral leaflets are more fragile. This is the reason why thicker mitral leaflets were more severely prolapsed. Thus, mitral valves in patients with mitral valve prolapse are relatively large, but they may be fragile. 


\section{Mitral regurgitation complicated with mitral valve prolapse}

In this study, all of mitral leaflet thickness, posterior mitral leaflet length and mitral annular diameter showed significant positive relation to the severity of mitral regurgitation complicated with mitral valve prolapse without ruptured chordae tendineae. This result strongly suggests that severe mitral regurgitation will tend to occur in larger mitral valves in patients with mitral valve prolapse.

Mitral annular dilatation was associated with mitral valve prolapse [12, 29, 36-41], and it was thought to be one of the causes of mitral regurgitation in patients with mitral valve prolapse [40]. However, the occurrence of mitral annular dilatation in MVP patients with minimal MR indicates primary or intrinstic annular dilatation rather than annular dilatation secondary to left atrial or left ventricular enlargement [41]. In this study, the RATIO did not show significant positive relation to the severity of mitral regurgitation, indicating that relative increase in mitral annular size is not the only cause of mitral regurgitation in MVP patients. Taking these data into consideration, we believe that mitral annular dilatation would be only partially responsible for mitral regurgitation occurring in patients with mitral valve prolapse.

It has been reported that the histological changes in the excised valves were, significantly different between patients with myxomatous mitral valve and ischemic mitral regurgitation [11]. So the myxomatous changes, in other words, thickenings of prolapsed mitral leaflets, would not be the result of mitral regurgitation but the cause of mitral regurgitation.

\section{Ruptured chordae tendineae}

Ruptured chordae tendineae was reported by Frothingham et al for the first time in 1934 [42]. Pomerance reported that ruptured chordae tendineae was thought to be preceded by mitral valve prolapse [43]. In this study, ruptured chordae tendineae occurred in patients with thicker or longer prolapsed mitral leaflets. This result confirms that ruptured chordae tendineae tends to occur in patients with severe mitral valve prolapse, and supports Pomerance's theory.

Tissue overlap may play a more significant role in the diastolic appearance of the flail mitral leaflet visualized by two-dimensional transesophageal echocardiography than do actual structural thickening of the leaflet tissue itself [28]. In this study, the prolapsed posterior mitral leaflets with ruptured chordae tendineae were thicker than those without ruptured chordae tendineae and those of the control subjects not only in diastole but also in systole.

\section{Clinical application}

The patients with mitral valve prolapse have usually benign clinical course. But, congestive heart failure due to progressive mitral regurgitation does occur [38]. Nishimura et al has reported that patients with thick mitral leaflets have belonged to the subset of high risk group of mitral valve prolapse using transthoracic M-mode echocardiography [22]. Our data show that thicker or longer mitral leaflets are predictors of severe mitral regurgitation. Patients with thicker or longer mitral leaflets belong to the subset of high risk group of mitral valve prolapse.

\section{Limitations}

Using transesophageal echocardiography we measured mitral leaflet thickness, mitral leaflet length and mitral annular diameter to assess the relation of mitral valve morphology to severe mitral regurgitation complicated with mitral valve prolapse. In this study most patients with severe mitral regurgitation had received vasodilators or diuretics. This pharmacotherapy would affect the size of the mitral annulus or the severity of the mitral regurgitation, but not the mitral leaflet thickness or length.

\section{Conclusions}

The leaflet thickness, leaflet length and annular diameter are "proportionally" redundant in patients with mitral valve prolapse, and the redundancy is closely related to the occurrence of ruptured chordae tendineae or severe mitral regurgitation. Measurement of mitral leaflet thickness or length by transesophageal echocardiography was very useful in identifying the high-risk group of mitral valve prolapse.

\section{References}

1. Levy D, Savage D. Prevalence and clinical feature of mitral valve prolapse. Am Heart J 1987; 113: 1291-8.

2. Devereux RB, Kramer-Fox R, Klingfield P. Mitral valve prolapse: causes, clinical manifestations, and management. Ann Intern Med 1989; 111: 305-17.

3. Wooley CF, Baker PB, Kolibash AJ, Kilman JW, Sparks EA, Boudoulas H. The floppy, myxomatous mitral valve, mitral valve prolapse, and mitral regurgitation. Prog 
Cardiovasc Dis 1991; 33: 397-433.

4. Boundous H, Kolibash AJ, Baker P, King B, Wooley CF. Mitral valve prolapse and the mitral valve prolapse syndrome: A diagnostic classification and pathogenesis of symptoms. Am Heart J 1989; 118: 796-818.

5. Fenster MS, Feldman MD. Mitral regurgitation: An overview. Curr Probl Cardiol 1995; 20: 193-280.

6. Roberts WC. Morphologic aspects of cardiac valve dysfunction. Am Heart J 1992; 123: 1610-32.

7. Kolibash AJ, Kilman JW, Bush CA, Ryan JM, Fontana ME, Wooley CF. Evidence for progression from mild to severe mitral regurgitation in mitral valve prolapse. Am J Cardiol 1986; 58: 762-7.

8. Pearson AC, St. Vrain J, Mrosek D, Labovitz AJ. Color Doppler echocardiographic evaluation of patients with a flail mitral leaflet. J Am Coll Cardiol 1990; 16: 232-239.

9. Jacobs W, Chamoun A, Stouffer GA. Mitral valve prolapse: A review of the literature. Am J Med Sciens 2001; 320: 401-10.

10. Hayek E, Gring CN, Griffin BP. Mitral valve prolapse. Lancet 2005; 365: 507-18.

11. Hickey AJ, Wilcken DEL, Wright JS, Warren BA. Primary (spontaneous) chordal rupture: relation to myxomatous valve disease and mitral valve prolapse. J Am Coll Cardiol 1985; 5: 1341-6.

12. Tresch DD, Doyle TP, Boncheck LI, Siegel R, Keeian $\mathrm{MH}$, Olinger GN, Brooks HL. Mitral valve prolapse requiring surgery. Clinical and pathological study. Am J Med 1985; 78: 245-50.

13. Jeresaty RM, Edwards JE, Chawla SK. Mitral valve prolapse and ruptured chordae tendineae. Am J Cardiol 1985; 55: 138-42.

14. Roberts WC, McIntosh CL, Wallace RB. Mechanisms of severe mitral regurgitation in mitral valve prolapse determined from analysis of operatively excised valves. Am Heart J 1987; 113: 1316-23.

15. Turri M, Thiene G, Bortolotti U, Mazzucco A, Gallucci V. Surgical pathology of disease of the mitral valve, with special reference to lesions promoting incompetence. Int J Cardiol 1989; 22: 213-9.

16. Nimura Y. Short history of the development of echocardiography with special reference to that in Japan (1). J Echocardiogr 2003; 1: 46-59.

17. Nimura Y. Short history of the development of echocardiography with special reference to that in Japan (2). J Echocardiogr 2004; 2: 27-35.

18. Marks AR, Choong CY, Sanfilippo AJ, Ferre M, Weyman AE. Identification of high-risk and low-risk subgroups of patients with mitral valve prolapse. N Engl J Med 1989; 320: 1031-6.

19. J Yoshikawa. Clinical Echocardiography The $2^{\text {nd }}$ edition: 113-4.
20. Sanfilippo AJ, Abdollah H, Burggraf GW. Quantization and significance of systolic mitral leaflet displacement in mitral valve prolapse. Am J Cardiol 1989; 64: 1349-55.

21. Chandraratna PAN, Nimalasuriya A, Duncan P, Rosin B, Rahimtoola SH. Identification of the increased frequency of cardiovascular abnormalities associated with mitral valve prolapse by two-dimensional echocardiography. Am J Cardiol 1984; 54 1283-5.

22. Nishimura RA, McGoon MD, Shub C, Miller FA Jr, Ilatrup DM, Tajik AJ. Echocardiographically documented mitral valve prolapse: long-term follow up of 232 patients. N Engl J Med 1985; 313: 1305-9.

23. Pini R, Greppi B, Kranmer-Fox R, Roman MJ, Devereux RB. Mitral valve dimensions and motion and familial transmission of mitral valve prolapse with and without mitral leaflet billowing. J Am Coll Cardiol 1988; 12: 142331.

24. Grayburn PA, Berk MR, Spain MG, Harrison MR, Smith MD, DeMaria AN. Relation of echocardiographic morphology of the mitral apparatus to mitral regurgitation in mitral valve prolapse: Assessment by Doppler color flow imaging. Am Herat J 1990; 119: 1095-102.

25. Zuppiroli A, Mori F, Favilli S, Barchielli A, Corti G, Montereggi A, Dolara A. Arrhythmias in mitral valve prolapse: Relation to anterior mitral leaflet thickening, clinical variables, and color Doppler echocardiographic parameters. Am Heart J 1994; 128: 919-27.

26. Weissman NJ, Pini R, Roman MJ, Kramer-Fox R, Andersen HS, Devereux RB, Spitzer MC. In vivo mitral valve morphology and motion in mitral valve prolapse. Am J Cardiol 1994; 73: 1080-8.

27. Freed LA, Benjamin EJ, Levy D, et al. Mitral valve prolapse in the general population. The benign nature of echocardiographic features in the Framingham Heart Study. J Am Coll Cardiol 2002; 40: 1298-304.

28. Louie EK, Langholz D, Mackin WJ, Wallis DE, Jacobs WR, Scanlon PJ. Transesophageal echocardiographic assessment of the contribution of intrinsic tissue thickness to the appearance of a thick mitral valve in patients with mitral valve prolapse. J Am Coll Cardiol. 1996; 28: 465-71.

29. Fukuda N, Oki T, Luchi A, et al. Predisposing factors for severe mitral regurgitation in idiopathic mitral valve prolapse. Am J Cardiol 1995; 76:503-7.

30. Malkowski MJ, Guo R, Orsinelli DA, Wooley CF, Tice FD, Pearson AC, Boudoulas H. The morphologic characteristics of frail mitral leaflets by transesophageal echocardiography. J Heart Valve Dis 1997; 6: 54-9.

31. Shrivastava S, Guthrie RB, EdwardsJE. Prolapse of the mitral valve. Mod Concepts Cardiovasc Dis. 1977; 46: 5761.

32. Davies MJ, Moore BP, Braimbridge MV. The floppy 
mitral valve. Study of incidence, pathology, complications in surgical, necropsy and forensic material. Br Heart J 1978; 40: 468-81.

33. Watanabe C, Sugiura M, Ohkawa S, et al. Pathology and histochemistry of mitral valve prolapse. J Cardiol 1993; 23: 69-77.

34. Tamura K, Fukuda Y, Ishizaki M, Masuda Y, Yamanaka N, Ferrans V. Abnormalities in elastic fibers and other connective-tissue components of floppy mitral valve. Am Heart J 1995; 129: 1149-58.

35. Pellerin D, Brecker S, Veyrat C. Degenerative mitral valve disease with emphasis on mitral valve prolapse. Heart 2002; 88 (Supple IV): iv-20-8.

36. Bulkley BH, Roberts WC. Dilatation of the mitral annulus. A rare cause of mitral regurgitation. Am J Med 1975; 59: 457-63.

37. Leachman RD, DeFrancheschi A, Zamalloa O. Late systolic murmurs and clicks associated with abnormal mitral valve ring. Am J Cardiol 1969; 23: 679-83.

38. Marchand P, Barlow JB, Duplessis LA, Webster I. Mitral regurgitation with rupture of normal chordae tendineae. Br Heart J 1966; 28: 746-58.

39. McKay R, Yacoub MH. Clinical and pathological findings in patients with "floppy" valves treated surgically. Circulation 1973; 48: suppl III-63-73.

40. Kay JH, Krohn BG, Zubiate P, Hoffman RL. Surgical correction of severe mitral prolapse without mitral insufficiency but with pronounced cardiac arrhythmias. J Thorac Cardiovasc Surg 1979; 78: 259-68.

41. Ormiston JA, Shah PM, Tei C, Wong M. Size and motion of the mitral valve annulus in man. II. Abnormalities in mitral valve prolapse. Circulation 1982; 65: 713-9.

42. Frothingham C, Hass GM. Rupture of normal chordae tendineae of the mitral valve. Am Heart J 1934; 9: 492-9.

43. Pomerance A. Ballooning deformity (mucoid degeneration) of atrioventricular valves. Br Heart J 1969; 31: 343-51. 